\title{
Ensayo \\ El concepto de cultura como fundamento de nuevos valores: una visión pluralista
}

\section{Resumen}

El presente ensayo desarrolla una reflexión sobre el concepto de cultura en el contexto contemporáneo definiendo nociones clave para la formulación de políticas públicas culturales en el siglo XXI como la identidad, la diversidad cultural y la interculturalidad. Asimismo, ofrece un marco conceptual básico sobre la cultura con una visión amplia y plural, ya que el concepto con el que se opere, se reflejará en las maneras de intervenir y determinará las lógicas que se deberán tener en cuenta a la hora de formular los lineamientos e implementar la política cultural.

\section{Palabras clave: cultura, identidad, diversidad cultural, interculturalidad, política cultural.}

\section{Abstract}

This essay develops a reflection on the concept of culture in the contemporary context defining key notions for the formulation of cultural public policies in the 21st century such as identity, cultural diversity and interculturality. It also offers a basic conceptual framework on culture with a broad and plural vision, since the concept with which it operates will be reflected in the ways of intervening and will determine the logics that should be taken into account when formulating the guidelines and implement cultural policy.

Palabras clave: culture, identity, cultural diversity, interculturality, cultural policy. 


\section{Introducción}

El estudio de cualquiera de las expresiones culturales del quehacer humano, y las formas de acción específicas para promover su desarrollo, debe comenzar por el análisis del concepto de cultura. Este es polisémico, se ha ido configurando en el tiempo a través de distintos enfoques y como resultado de diferentes procesos por los que han atravesado las sociedades. Las reflexiones en torno a aquella han cobrado mayor importancia en los últimos años dentro del ámbito académico, político y en la agenda de los organismos internacionales ${ }^{1}$. Junto a otros valores específicos como la identidad, la diversidad cultural y la interculturalidad, la cultura ha sido objeto de estudio y debate en investigaciones académicas, documentos regionales e internacionales, incluso, se ha incorporado en algunas constituciones latinoamericanas².

Este ensayo sostiene la afirmación de que a partir de la cultura, en su sentido más amplio, es posible desarrollar procesos para transformar la sociedad, crear valores, fomentar una vida más libre, construir identidades propias, reconocer y respetar la diversidad cultural y crear relaciones interculturales. Sus diversas manifestaciones desempeñan un importante papel en la sociedad contemporánea.

Un punto de partida para esta reflexión es que la formulación e implementación de las políticas culturales están asociadas a la noción de cultura, ya que, en dependencia del concepto que se asuma, se reflejará la manera en que se opere y determinará las lógicas atendidas durante la formulación de los lineamientos e implementación de una política cultural específica.

\section{La cultura: un concepto plural}

Las reflexiones sobre la cultura han adquirido importancia en los últimos años en varios campos y específicamente en las políticas públicas, a pesar de las múltiples contribuciones desde varios sectores para la elaboración de una definición. Hasta el momento no existe un consenso de todas estas reflexiones, sobre todo a la hora de articular estos enfoques con las políticas culturales.

La UNESCO (1982) define a la cultura como "el conjunto de los rasgos distintivos, espirituales y materiales, intelectuales y afectivos que caracterizan a una sociedad o un grupo social. Ella engloba, además de las artes y las letras, los modos de vida, los derechos fundamentales al ser humano, los sistemas de valores, las tradiciones y las creencias".

Las características y nuevos procesos sociales, económicos y tecnológicos generados en el mundo contemporáneo, reclaman un concepto de cultura que abarque múltiples dimensiones y tome en cuenta la reflexión académica y a sus voces más autorizadas, y que, además de un carácter patrimonial y estético, la doten de un sistema diferente de percepción, valores y

$1 \quad$ Organización de las Naciones Unidas para la Educación, la Ciencia y la Cultura (UNESCO); Organización de Estados Iberoamericanos (OEI); Banco de Desarrollo Iberoamericano (BID); Mercado Común del Sur (MERCOSUR); Convenio Andrés Bello (CAB), Alianza Bolivariana para los Pueblos de Nuestra América-Tratado de Comercio de los Pueblos (ALBA-TCP).

$2 \quad$ Colombia, 1991; México, 1992; Brasil, 1998 y Ecuador, 2008. 
significados relacionados con procesos de diversa índole.

Desde el ámbito académico, no pocos autores se han mostrado preocupados por definir un concepto de cultura que responda a los procesos históricos en que vivimos. Ello ha generado un debate que pone de manifiesto las múltiples facetas y el importante papel que desempeña tal noción en las sociedades.

La cultura puede ser abordada a través de dos registros que se complementan entre sí: el estético y el antropológico (Miller y Yúdice, 2004), en los que confluyen las artes, los modos de vida, la interacción con otras culturas, los modos de organización social (UNESCO, 1982). La cultura, en primer lugar, implica pertenencia y tiene la función de diferenciar a un grupo de otro (Giménez, 2012); pero también es necesario entenderla como una estrategia para el desarrollo (Martinell, 2015), dado que representa un recurso para atraer inversiones, generar crecimiento económico y empleos (Canclini, 2005), así como innovación tecnológica y transformación social (Bustamante, 2011).

Según Martín-Barbero (2012), la cultura puede ser estudiada como un modelo de comportamiento, un conjunto de gramáticas axiológicas o de sistemas narrativos. La cultura está conformada por identidades que forman parte de la diversidad cultural, y la diversidad, entendida como recurso y oportunidad, es el principio rector de la cultura (Zapata, 2016).

Finalmente, la cultura "es un recurso global y territorial que cimienta el tejido social de una sociedad, su identidad, su cohesión y su nivel de desarrollo" (Gobierno Vasco, 2016, s/p).

Hoy se ha comenzado a comprender la interrelación de la cultura con lo que anteriormente se consideraba únicamente espacio político o económico. Por esa razón, la cultura se considera un recurso estratégico cuyas expresiones dan lugar a transformaciones sociales, humanas y económicas, y contribuye al desarrollo y a la sostenibilidad (Yúdice, 2002). Desde esta perspectiva, la cultura es un importante recurso para el desarrollo.

En las últimas décadas se viene demostrando el impacto que la cultura tiene sobre la economía y el desarrollo. Tanto las estadísticas como las investigaciones en otras áreas del conocimiento evidencian el aporte que tiene la cultura al Producto Interno Bruto (PIB) y a las plusvalías que genera el patrimonio cultural, el turismo, las industrias culturales y creativas. Es reconocida su contribución a la lucha contra la pobreza y la exclusión, a la regeneración urbana, a la mejora de la calidad de vida y a la cohesión social. Vich (2012) la ubica como un agente clave para el cambio social.

A partir del análisis de los diferentes criterios aportados por los autores de referencia, en este ensayo se asume la cultura en un sentido epistémico, como los diferentes rasgos, valores y manifestaciones del ser humano, en los que confluyen las artes, los modos de vida, la interacción entre los diferentes grupos que conforman la sociedad, las distintas formas de organización social y recurso para la transformación y el desarrollo.

De la misma manera, nuestra visión de cultura está en relación con una serie de representaciones 
como la identidad, la diversidad y la interculturalidad, ya que como señala Mato (2001) estas construcciones sociales, en tiempos de globalización, tienen una importancia fundamental en la constitución de los actores sociales ${ }^{3}$ - en las que se involucran a dimensiones como la política-, y en la manera de relacionarse.

\section{Las identidades en tiempos de globalización}

La identidad es esencial para comprender el mundo contemporáneo. Es un concepto que se construye desde patrones de reconocimiento y diferenciación de elementos de base cultural hacia una colectividad. Está sujeta a transformaciones en el tiempo, ya que posee valor cambiante dada su continua interacción con el exterior.

Giménez (2012) sostiene que las identidades se construyen a partir de la apropiación por parte de los actores sociales de determinados repertorios culturales fuera de su contexto y en la propia unidad. Estas características se desarrollan en lógicas de inclusión y exclusión. Para MartínBarbero (2002) la identidad puede ser pensada tanto como raíz, territorio y memoria como en redes, flujos o migraciones; a la vez, la relaciona con trayectorias y relatos, pues sostiene que la pluralidad cultural es reconocida cuando la diversidad de identidades puede ser narrada a por medio de relatos.

En las sociedades contemporáneas los relatos identitarios pasan a ser producto de culturas heterogéneas, espacio en el cual surgen nuevos sujetos, identidades y espacios híbridos, en los que se intenta reivindicar el derecho a la memoria y a los territorios para llenar lo que Stuart Hall denomina "vacío identitario" provocado por los nuevos flujos territoriales, comunicacionales y migratorios.

Si en la década de los ochenta el debate se centraba en cómo proteger la identidad nacional a través de políticas culturales, en la actualidad los estudios culturales conocidos como la dirigen la mirada hacia el otro y resustancializan las identidades poscoloniales que están surgiendo después de la crisis de los Estados nación.

Estas nuevas identidades no tienen fronteras, los sujetos que conforman los grupos o comunidades son parte de nuevas culturas donde sus identidades se constituyen como "puntos de sutura" (Hall, 1996, p. 5). Las identidades hoy en día son transterritoriales y, como señala MartínBarbero (2001), están conformadas por imaginerías nacionales, tradiciones locales y flujos de información transnacionales, que generan un nuevo modelo de ciudadanía y nuevos modos de estar juntos.

Estos factores dan lugar a imaginarios donde los intercambios entre los actores que conforman las sociedades desencadenan la asunción del reconocimiento del yo y de los otros, lo que implica la afirmación de una identidad determinada. Si no existe ese reconocimiento de la diferencia, el intercambio resulta fallido.

3 La conformación de estos actores sociales, como movimiento, surge en los años ochenta bajo el lema del multiculturalismo. Nacen como un conjunto heterogéneo de movimientos sociales conformado por varios grupos

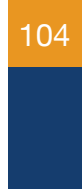


Para dar visibilidad a esa pluralidad de culturas existentes en una sociedad, y que se manifiestan a través de lenguajes tradicionales y otros más contemporáneos, es necesario el reconocimiento de las diferentes identidades que conforman un colectivo y su diversidad.

\section{El giro positivo de la diversidad cultural y su enfoque en relación con los cambios} culturales

Las sociedades actuales se caracterizan por ser complejas, diversas, interculturales e interconectadas. Los procesos de globalización asociados a las lógicas del mercado y a las tecnologías digitales han desdibujado las fronteras simbólicas. Las identidades y las culturas se nutren de la interacción real y virtual y se van redefiniendo constantemente. Ese contexto reclama el reconocimiento del otro y el respeto por las diferentes diversidades, ya sean étnicas, culturales o de las nuevas categorías identitarias que han sido reconocidas en los últimos años, y logrado cierta legitimidad en las sociedades actuales, tales como género y diversidad sexual. Otras son más frágiles y transitorias constituidas a partir de los procesos de comunicación global, el consumo, la edad, el nickname, etc. (Nivón, 2013).

La diversidad cultural - multiplicidad de formas en que se expresan las culturas de los grupos y sociedades - tiene que ver con la idea del otro y con la protección y promoción de los grupos minoritarios. Es una noción conformada por las diferencias y semejanzas de los distintos componentes que engloba "que no son solo raciales, sino que entrañan también cuestiones de clases sociales, relaciones económicas, de género, lingüísticas, políticas, culturales, religiosas, etc. Y también relaciones de poder y justicia social” (Olmos, 2008, p. 38).

La diversidad es un elemento constitutivo de la cultura, su definición le ofrece un perfil antropológico a la cultura, en el que están contenidas la heterogeneidad y pluralidad de las identidades, grupos, sociedades, culturas y la manera de interactuar entre cada una. Se ha convertido en una opción a las divergencias de las identidades y a los Estados nación, que priorizaron la homogeneidad a costa de la diversidad y la diferencia (Ochoa, 2008). Hoy, la diversidad reclama el respeto y el reconocimiento de esas diferencias, para lograr alternar la perspectiva propia con la de los otros.

En las sociedades actuales es recurrente hablar de culturas, en plural. Las fronteras simbólicas se han desdibujado, hasta los mercados han perdido sus fronteras (Appudarai, 2014), las sociedades actuales presentan nuevas formas de desigualdad y dan lugar a conflictos culturales de nuevo orden. Las lógicas de la globalización, los flujos migratorios, los proyectos transnacionales, las fronteras reales o virtuales, por solo mencionar algunas categorías que conforman el escenario cultural de los últimos años, son influencias externas que proponen expresiones inéditas de interacción entre culturas.

En estas sociedades es importante reconocer la diversidad cultural, no solo a partir de las

minoritarios invisibilizados: indígenas, afros, gay-lesbianas, etc. Estos movimientos contestatarios buscaron elaborar una identidad propia y reivindicar el valor de la "diferencia" étnica o cultural, los derechos humanos, la biodiversidad, asuntos de género y opciones sexuales (Ochoa, 2008). 
condiciones étnicas, sino a través de las múltiples identidades que son parte del territorio con estrategias destinados a las minorías y a los grupos discriminados, de manera que se logre un ambiente incluyente, que ayude a visibilizar las identidades, defender sus derechos individuales y colectivos. Además de apoyar el derecho a la diferencia de los grupos que puedan quedar fuera de las normas sociales, culturales y de las nuevas identidades (Nivón, 2013). Su reconocimiento se ha convertido en un postulado político, gran parte, por la acción de estos grupos que luchan por entrar a la esfera pública para reorientar la política pública y que exigen un cambio de la homogeneidad a la diversidad como nueva norma social.

La diversidad cultural ofrece un marco de herramientas conceptuales para articular nuevas políticas culturales. El desafío de las sociedades contemporáneas consiste, entonces, en replantear las fórmulas tradicionales de las políticas, para que se ajusten a los nuevos escenarios.

\section{La interculturalidad como estrategia para la interacción cultural y el reconocimiento}

\section{del otro}

La noción de interculturalidad ha surgido con fuerza en las últimas décadas. Específicamente surgió como respuesta al multiculturalismo, el cual estuvo caracterizado por reconocer la diversidad y por admitir la tolerancia entre las culturas; pero, no promovió el diálogo y el respeto entre ellas.

La interculturalidad, por el contrario, al estimular la interacción cultural interfiere directamente en las prácticas sociales, involucrando varias dimensiones, tales como la política y propiciando procesos de negociación, intercambio de saberes y la construcción social con el objetivo de reconocer y valorar al otro (García Canclini, 2004).

La discusión sobre la interculturalidad ha girado en torno a la necesidad de reconocimiento de las distintas maneras de diversidad cultural. En este sentido, la cultura se ha convertido en una de las formas de organizar la diversidad, y la interculturalidad en una opción para generar interacción e intercambio entre las diferentes culturas o grupos existentes dentro de una sociedad.

Varios autores coinciden en la interculturalidad permite organizar de manera sostenible la pluralidad cultural de las sociedades, la relacionan con la identidad y como un recurso para la visibilidad y la protección de grupos diversos dentro del espacio público, cuyo objetivo fundamental consiste en favorecer la interacción cultural y fomento de expresiones culturales compartidas por medio del diálogo y entendimiento mutuo en ambientes inclusivos.

La estrategia intercultural para el intercambio tiene que ver con "que cada cultura tiene que enfrentarse cuerpo a cuerpo a las otras culturas, es decir, que una cultura es reconocida en la medida en que es traducible y traducida. Y es capaz de traducir a otras" (Martín-Barbero, 2008, p. 18), este intercambio a través de procesos verbales y no verbales supone la mejor estrategia para gestionar la diversidad en las sociedades contemporáneas, ya que el entendimiento y reconocimiento del otro pone en manifiesto las posibilidades que tiene la interculturalidad 
como herramienta de diálogo, inclusión, cohesión social y su aporte para la construcción de sociedades más democráticas.

\section{Conclusiones}

El objetivo de este ensayo consiste en exponer el debate actual sobre la cultura y construir un marco que garantice su desarrollo e intervenciones estatales en materia de cultura más inclusivas. Otro eje de discusión en este trabajo tiene que ver con las nociones de identidad, diversidad cultural e interculturalidad, con el fin de mostrar las ventajas que tienen a la hora de incluirlas en la dimensión cultural y política a partir de las reflexiones de las voces más autorizadas en la materia.

El concepto de cultura en la actualidad es amplio y se ha expandido hacia distintos campos como la política, la economía, lo social, lo tecnológico, al respecto, se intenta ofrecer un horizonte teórico con una visión amplia y plural del término que sirva de sustento a la hora de formular e implementar políticas culturales.

Las interrogantes sobre la identidad, la diversidad cultural y la interculturalidad constituyen categorías imprescindibles a la hora de formular políticas culturales que aspiren a reconocer las identidades múltiples existentes en los territorios, administrar eficientemente la diversidad cultural, promover el diálogo, impulsar la creatividad y propiciar la equidad e igualdad social en un proyecto político con un carácter trasformador y a largo plazo. 


\section{Referencias bibliográficas}

Bustamante, E. (2011). Cultura digital: La nueva cultura clásica. TELOS. Cuadernos de Innovación y comunicación, (88), 59-64.

García Canclini, N. (2004). Diferentes, desiguales y desconectados. Mapas de la interculturalidad. Barcelona, España: Gedisa Editorial.

García Canclini, N. (2005). Todos tienen cultura. ¿Quién puede desarrollarla? Presentado en Seminario sobre Cultura y Desarrollo, Banco Interamericano de Desarrollo, Washington. Recuperado de http://www.congresoed.org/wp-content/uploads/2014/10/Canclini-Cultura-desarrollo.pdf

Giménez, G. (2012). La cultura como identidad y la identidad como cultura. Recuperado de https://estudioscultura.wordpress.com/2012/03/13/gilberto-gimenez-la-cultura-como-identidad-y-la-identidad-como-cultura/

Gobierno Vasco (2016). Informe 2015. Análisis de la cadena de valor y propuestas de política cultural (Primer informe sobre el estado de la cultura vasca. CAE 2015). Vitoria.

Hall, S. y Du Gay, P. (Comps). (1996). Cuestiones de identidad cultural. Buenos Aires, Argentina: Editorial Amorrortu.

Martín-Barbero, J. (2002). La globalización en clave cultural: una mirada latinoamericana. Presentado en Coloquio internacional Globalismo y pluralismo, Montreal. Recuperado de https://www.scribd.com/document/6315058/La-globalizacion-en-clave-cultural-Una-mirada-latinoamericana

Martín-Barbero, J. (2008). Diversidad cultural y convergencia digital. I/C - Revista científica de Información y comunicación, (5), 15-25.

Martín-Barbero, J. (2012). De la Comunicación a la Cultura: perder el "objeto" para ganar el proceso. Signo y pensamiento, $X X X(60), 76-84$.

Martinell, A. (2015). Cultura y desarrollo: Reflexión sobre las nuevas funciones de las políticas culturales territoriales. En M. Rojas, La gestión cultural en 3 D: debates, desafíos y disyuntivas (pp. 33-47). Santiago de Chile: Fondo de Cultura Económica.

Mato, D. (2001). Producción transnacional de representaciones sociales y transformaciones sociales en tiempo de globalización. En D. Mato, Estudios Latinoamericanos sobre cultura y transformaciones sociales en tiempos de globalización (pp. 127-159). Buenos Aires, Argentina: CLACSO.

Mato, D. (2009). Contextos, conceptualizaciones y usos de la idea de interculturalidad. En M. Aguilar, E. Nivón, A. Portal, y R. Winocur, Pensar lo contemporáneo: De la cultura situada a la convergencia tecnológica (pp. 28-50). Barcelona, España: Antrhopos. 
Miller, T., y Yúdice, G. (2004). Política cultural. Barcelona, España: Gedisa Editorial.

Nivón, E. (2013). Las políticas culturales en América Latina en el contexto de la diversidad. En Hegemonía cultural y políticas de la diferencia (pp. 23-45). Buenos Aires: CLACSO.

Ochoa, A. M. (2008). Desencuentros entre los medios y las mediaciones: Estado, diversidad y políticas de reconocimiento cultural en Colombia. En Lacarrieu, M. \& Álvarez, M. (Comp.), La (Ind)Gestión cultural. Una cartografía de los procesos culturales contemporáneos (pp. 153-174). Buenos Aires, Argentina: La Crujía Ediciones.

Olmos, A. H. (2008). Cultura. Estrategia para el desarrollo local. Madrid, España: AECID.

Organización de las Naciones Unidas para la Educación, la Ciencia y la cultura (UNESCO). (1982). Mondiacult. Recuperado de http://www.culturalrights.net/descargas/drets_culturals400.pdf

Organización de las Naciones Unidas para la Educación, la Ciencia y la cultura (UNESCO). (2005). Convención para la promoción y protección de las expresiones culturales. Recuperado de http://unesdoc.unesco.org/ images/0014/001429/142919s.pdf

Vich, V. (2012). Desculturizar la cultura. Recuperado de http://noticias.universia.pr/tiempo libre/noticia/2011/05/11/819680/desculturizar-cultura-retos-actuales-politicas-culturales.pdf

Yúdice, G. (2002). El recurso de la cultura. Usos de la cultura en la era global. Barcelona, España: Gedisa Editorial.

Zapata-Barrero, R. (2016). Políticas culturales e interculturalidad. Barcelona, España: Ediciones Bellaterra. 